\title{
IIR Ultra-Wideband Pulse Shaper Design
}

\author{
Chun-Yang Chen and P. P. Vaidyanathan \\ Dept. of Electrical Engineering, MC 136-93 \\ California Institute of Technology, Pasadena, CA 91125, USA \\ E-mail: cyc@caltech.edu, ppvnath@systems.caltech.edu
}

\begin{abstract}
This paper introduces a new method to generate an ultra-wideband (UWB) pulse to fill up the Federal Communications Commission (FCC) mask. One way to generate the pulse is by summing a weighted delayed elementary waveform (e.g., a Gaussian monocycle pulse). This method can be reduced to an FIR filter design problem. In this paper, an IIR-like method is introduced. It generates the UWB pulse using the weighted sum of not only the delayed elementary waveform but also the feedback waveforms. Allpass decomposition and lattice structures are used to reduce the complexity of the implementation. Simulation shows that the IIR based method has higher filling efficiency than the FIR based method. Simulation also shows that the impulse response of the IIR shaper decays very fast. Therefore the expected long transient created by the IIR shaper is negligible. ${ }^{1}$
\end{abstract}

\section{INTRODUCTION}

Ultra-Wideband (UWB) communication technology allows wireless communication at very high speed data rates across short distances with a low-power source [1]. Due to its attractive features, UWB systems have potential for many applications such as indoor wireless data network. In 2002, the Federal Communications Commission (FCC) approved a spectral mask for operation of UWB devices [2]. It allows a huge bandwidth $(3.1 \mathrm{GHz}-10.6 \mathrm{GHz})$. Because the extremely broad bandwidth overlaps the coexistent RF transmission, the FCC has imposed restriction on the equivalent isotropic radiated power (EIRP) spectrum of the UWB signal. The FCC power spectrum mask $M(f)$ is shown in Fig. 1. The UWB pulse spectrum is required to be within this mask. It has been proposed that impulse radio systems (which transmit very short pulses without using RF carriers) are good candidates to satisfy these constraints. In order to utilize the bandwidth allowed by the FCC, the pulse should be designed such that the radiated power is maximized while satisfying the FCC constraint. Previous work on pulse shaper design can be found in [3], [4] and [5].

The EIRP spectrum can be expressed as

$$
S_{E I R P}(f) \quad\left|H_{e q}(f)\right|^{2} S_{m}(f)|P(f)|^{2},
$$

where $P(f)$ is the Fourier transform of the pulse, $S_{m}(f)$ depends on the modulation and $H_{e q}(f)$ is the transfer function from modulated pulse train to the radiated electromagnetic field on which the EIRP mask is imposed. Therefore, the FCC mask limitation is

$$
\left|H_{e q}(f)\right|^{2} S_{m}(f)|P(f)|^{2} \leq M(f) .
$$

\footnotetext{
${ }^{1}$ Work supported in parts by the NSF grant CCF-0428326 and the California Institute of Technology
}

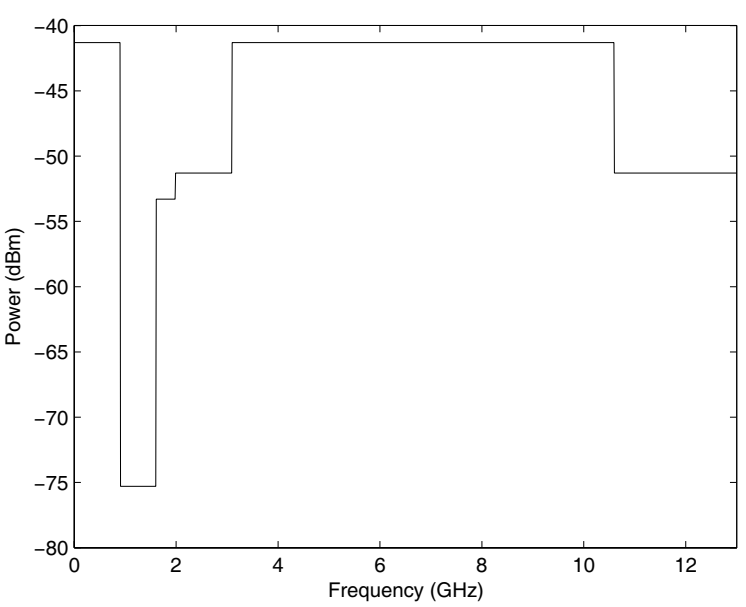

Fig. 1. The FCC mask for UWB communication.

In order to utilize the bandwidth allowed by mask $M(f)$, the transmitted power should be maximized under the FCC limitation. The pulse shape $p(t)$ can be optimized for this. So the optimization problem is:

$$
p(t) \int_{F_{\min }}^{F_{\max }}\left|H_{e q}(f)\right|^{2} S_{m}(f)|P(f)|^{2} d f
$$$$
\text { subject to }\left|H_{e q}(f)\right|^{2} S_{m}(f)|P(f)|^{2} \leq M(f) \text {. }
$$

Typically one takes $F_{\min } \quad$, and $F_{\max } \quad \mathrm{GHz}$. To evaluate the efficiency of the UWB pulse, the mask-filling efficiency $\eta$ is defined in [3] by

$$
\eta \frac{\int_{F_{\min }}^{F_{\max }}\left|H_{e q}(f)\right|^{2} S_{m}(f)|P(f)|^{2} d f}{\int_{F_{\min }}^{F_{\max }} M(f) d f} .
$$

This is a measure of how closely the spectrum fits Fig. 1, while remaining within the mask. Without any other constraints on $p(t)$, the ideal solution to this optimization problem is

$$
|P(f)|^{2} \quad \frac{M(f)}{\left|H_{e q}(f)\right|^{2} S_{m}(f)},
$$

which achieves filling efficiency $\eta$. In practice, we cannot generate waveforms with arbitrary $P(f)$ with analog circuits. However, a practical way to design the UWB pulse is to approximate this ideal solution using the available waveforms. In [3], [4], the UWB pulse generator is a zero-order digital to 
analog converter (DAC) which generates a signal in the form

$$
p(t) \quad \sum_{n=0}^{M} b_{n} g(t-n T),
$$

where $g(t)$ is some elementary waveform which can be generated by analog circuitry such as the Gaussian monocycle pulse. The choice of $T$ will be discussed later. In [3], a global search algorithm is used to maximize the filling efficiency $\eta$. In [4], the Parks-McClellan algorithm is used to design the coefficients $\left\{b_{n}\right\}$ so that the frequency response of the pulse $P(f)$ approximates the ideal solution in Eq. (2).

Eq. (3) can be viewed as an FIR filter $\left\{b_{n}\right\}$ acting on continuous waveform $g(t)$. It is well know that with the same complexity, IIR filters have better frequency responses than FIR filters. In this paper, instead of using the FIR based method in Eq. (3), we proposed a new way to generate the pulse by an IIR based method. Experiments show that with the same complexity, our method has better filling efficiency than the FIR based method.

\section{Previous WORK}

In this section, we will briefly explain what has been done in [4] and [3]. In [4], the Parks-McClellan algorithm is used to design the pulse shaper. The frequency response of the pulse generated using FIR based method in Eq. (3) can be expressed as

$$
P(f) \quad \sum_{n=0}^{M} b_{n} e^{-i \frac{2 \pi n}{T} f} G(f), F_{\min } \leq f \leq F_{\max },
$$

where $G(f)$ is the frequency response of the elementary waveform $g(t)$. In [4], $g(t)$ is the Gaussain monocycle pulse. That is

$$
g(t) \quad \sqrt{e} \frac{t}{\tau_{g}} e^{-2\left(\frac{t}{\tau_{g}}\right)^{2}},
$$

where $\tau_{g}$ represents its peak amplitude. To approximate the ideal solution in Eq. (2), one can choose $\left\{b_{n}\right\}$ so that

$$
\begin{aligned}
& \left|\sum_{n=0}^{M} b_{n} e^{-i \frac{2 \pi n}{T} f}\right|^{2}\left|H_{e q}(f)\right|^{2} S_{m}(f)|G(f)|^{2} \approx M(f), \\
& F_{\min } \leq f \leq F_{\max }
\end{aligned}
$$

is satisfied. This reduces the problem to an FIR filter design problem. In [4], the authors uses the Parks-McClellan algorithm to design the $M+$-tap filter $\left\{b_{n}\right\}$. This algorithm is optimal in the sense that the maximum approximation error is minimized. Note that $\sum_{n=0}^{M} b_{n} e^{-i \frac{2 \pi n}{T} f}$ is periodic in $f$ with period $\frac{1}{T}$. However, as long as $T$ is small enough so that there are no repetitions in the region $F_{\min } \leq f \leq F_{\max }$, we can design $\left\{b_{n}\right\}$ appropriately. In [4], the typical values $T$ ps and $T \quad$ ps are used. For $T$. ps, the sampling rate is $/$. ps $\mathrm{GHz}$. We get full band control over $\mathrm{GHz} \leq f \leq \mathrm{GHz}$. For $T \quad$ ps, the sampling rate is $/$ ps . GHz, so the frequency response in the range $\mathrm{GHz} \leq f \leq$. $\mathrm{GHz}$ can be specified. Because $\left\{b_{n}\right\}$ are real, the magnitude response is symmetric around $6.85 \mathrm{GHz}$. If the EIRP spectrum is under the mask for $0 \mathrm{GHz} \sim 6.85 \mathrm{GHz}$, it will be also under the mask for $6.85 \mathrm{GHz} \sim 13.7 \mathrm{GHz}$ because of symmetry. This leads to a suboptimal design. However, by thus increase $T$, the number of filter taps can be reduced significantly.

In [3], the same FIR based structure in Eq. (3) is adopted. However, more practical considerations are taken into account. The transfer function from modulated signal to radiated electromagnetic field, $H_{e q}(f)$ is actually considered. The coefficients $\left\{b_{n}\right\}$ are chosen from a finite set $S$ restricted to the hardware implementation instead of real numbers. The filling efficiency mentioned in Eq. (1) is developed. Then an exhaustive search for $\left\{b_{n}\right\}$ that has the highest efficiency $\eta$ in $S$ is implemented. The following optimization:

$$
\begin{array}{r}
\left\{b_{n}\right\} \in S, a_{c} \\
\text { subject to } a_{c}\left|H_{e q}(f)\right|^{2} S_{m}(f)|P(f)|^{2} \leq M(f)
\end{array}
$$

is done, where $a_{c}$ is a scaling factor such that the EIRP spectrum is under $M(f)$. These papers use the FIR based method to generate the pulse. They inspire us to think of generating pulses by an IIR based method.

\section{IIR ULTRA-WIDEBAND PULSE SHAPER}

Instead of using the FIR based method in Eq. (3), we propose a new way to generate the pulse by using the equation

$$
p(t) \quad \sum_{n=0}^{M} b_{n} g(t-n T)-\sum_{n=1}^{N} a_{n} p(t-n T),
$$

where $g(t)$ is some elementary waveform like the Gaussian monocycle pulse. This can be implemented by the scheme in Fig. 2. This is analogous to recursive difference equations,

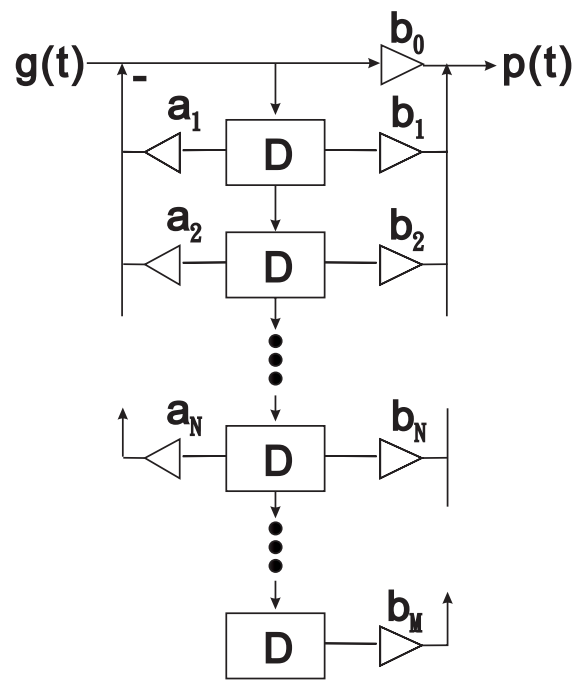

Fig. 2. The scheme for generating the pulse $p(t)$ recursively. Here "D" denotes one unit of analog delay.

hence the name "IIR method". In this case, the Fourier 
transform of the pulse is

$$
P(f) \quad \frac{\sum_{n=0}^{M} b_{n} e^{-i \frac{2 \pi n}{T} f}}{\sum_{n=0}^{N} a_{n} e^{-i \frac{2 \pi n}{T} f}} G(f),
$$

where $G(f)$ is the Fourier transform of $g(t)$ and $a_{0}$ for convenience. To approximate the ideal solution in Eq. (2), the power spectrum needs to fit within the mask. One can choose $\left\{a_{n}\right\}$ and $\left\{b_{n}\right\}$ so that

$$
\begin{aligned}
& \left|\frac{\sum_{n=0}^{M} b_{n} e^{-i \frac{2 \pi n}{T} f}}{\sum_{n=0}^{N} a_{n} e^{-i \frac{2 \pi n}{T} f}}\right|^{2}\left|H_{e q}(f)\right|^{2} S_{m}(f)|G(f)|^{2} \approx M(f), \\
& F_{\min } \leq f \leq F_{\max }
\end{aligned}
$$

This reduces the problem to an IIR filter design problem. However, the magnitude response of the filter is not simply highpass or lowpass. It needs to fit to a specific curve controlled by $\left|H_{e q}(f)\right|^{2} S_{m}(f)|G(f)|^{2}$ and the mask $M(f)$. Unlike the FIR case, there is no standard technique to design an IIR filter to fit an arbitrary magnitude response. To solve this, we divide the design problem into two parts. We first design an IIR equiripple filter $H_{1}(z)$ and then design an FIR filter $H_{2}(z)$ by the Parks-McClellan algorithm. The overall IIR filter $H_{1}(z)$ is $H(z) \quad H_{1}(z) H_{2}(z)$. The IIR filter has a relatively sharper transition band, therefore we use it to fit the transition band of $M(f)$. It is designed to have passband over . $\mathrm{GHz} \leq f \leq$. GHz. That is

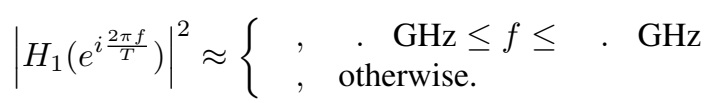

On the other hand, after $H_{1}(z)$ is determined, an FIR filter $\mathrm{H}_{2}(z)$ is used to provide the flexibility to compensate the nonflat passband caused by $\left|H_{e q}(f)\right|^{2} S_{m}(f)|G(f)|^{2}$. This can be done by solving the approximation problem

$$
\begin{array}{r}
\left|H_{2}\left(e^{i \frac{2 \pi f}{T}}\right)\right|^{2}\left|H_{1}\left(e^{i \frac{2 \pi f}{T}}\right)\right|^{2}\left|H_{e q}(f)\right|^{2} S_{m}(f)|G(f)|^{2} \approx M(f), \\
F_{\min } \leq f \leq F_{\max }
\end{array}
$$

This approximation can be done by the Parks-McClellan algorithm. By this separated design, we obtain both of the advantages of IIR equiripple filter and FIR minimax filter. Sharp transition band and flexibility to fit arbitrary magnitude response are obtained. Thus the filling efficiency can be increased under the same complexity.

\section{DESIGN EXAMPLES}

Three pulse design examples are compared in this section. The first example is a reproduction of [4]. The pulse is generated by the FIR based method in Eq. (3) and the Parks-McClellan algorithm is used to design the coefficients $\left\{b_{n}\right\}$. The second example is the pulse generated by the IIR based method in Eq. (5) and a standard IIR filter is used to obtained the coefficients $\left\{b_{n}\right\}$ and $\left\{a_{n}\right\}$. The third example is the pulse generated by the IIR based method in Eq. (5) with the design procedure we described in Section III. We first use a standard elliptic IIR filter to fit the transition band of the mask and then use a minimax FIR filter to compensate the nonflat passband caused by the Gaussian monocycle pulse and the transfer function $\left|H_{e q}(f)\right|^{2} S_{m}(f)$.

The parameters are listed in the following:

1) In order to compare the three cases under fixed cost, the number of multiplications in Fig. 2. is fixed to be 17.

2) The duration $T$ in Eq. (5) is chosen to be 73ps. In this case, the sampling rate is $1 / 73 \mathrm{ps}=13.7 \mathrm{GHz}$, so the frequency response in the range $\mathrm{GHz} \leq f \leq$. $\mathrm{GHz}$ can be specified. Because the signals are real, the magnitude response in the range. $\mathrm{GHz} \leq f \leq$. $\mathrm{GHz}$ is symmetric to that in the range $\mathrm{GHz} \leq f \leq$ . GHz.

3) The parameter $\tau_{g}$ in the Gaussian monocycle pulse is chosen to be 50ps. This choice makes the magnitude response of the Gaussian monocycle pulse have its peak value around $6.85 \mathrm{GHz}$.

4) In the following example, we assume $H_{e q}(f)$ and $S_{m}(f)$, as in [4]. These assumptions can be removed with appropriate modification of the design procedure.

Fig. 3 shows a comparison of the results obtained using these three methods.

A. FIR case [4]. The pulse is generated by FIR based method. The Parks-McClellan algorithm [6] is used to find $\left\{b_{n}\right\}$ so that Eq. (4). is satisfied. This can be viewed as a special case of the IIR scheme with $a_{0} \quad$ and $a_{n}$ for $n /$. To fix the number of multiplications to 17 , we choose the order $M$. The pulse power spectrum is shown in Fig. 3A. The filling efficiency $\eta$. $\%$.

B. Elliptic IIR filter. The pulse is generated by the IIR based method in Eq. (5). The standard elliptic IIR filter design is used to find $\left\{a_{n}\right\}$ and $\left\{b_{n}\right\}$ so that

$$
\left|\frac{\sum_{n=0}^{M} b_{n} e^{-i \frac{2 \pi n}{T} f}}{\sum_{n=0}^{N} a_{n} e^{-i \frac{2 \pi n}{T} f}}\right| \approx\left\{\begin{array}{l}
, \quad \mathrm{GHz} \leq f \leq . \mathrm{GHz} \\
, \quad . \mathrm{GHz} \leq f \leq . \quad \mathrm{GHz} .
\end{array}\right.
$$

Because the magnitude response is symmetric around 6.85 $\mathrm{GHz}$, it also has passband in the range . $\mathrm{GHz} \leq f \leq$. GHz. Therefore, the overall passband is . GHz $\leq f \leq$. GHz. We choose the orders $M \quad N \quad$. Because of the symmetry of the numerator $\left\{b_{n}\right\}$, the number of multiplications in the numerator is 6 . The total number of multiplications is therefore 16. The pulse power spectrum is shown in Fig. 3B. Because of the nonflatness of $G(f)$, the passband of the pulse is not flat. This seriously decreases the filling efficiency. The filling efficiency $\eta \quad \%$ in this case.

C. The new method of Section III. The pulse is generated by the IIR based method in Eq. (5). The design procedure described in Section III is applied. We first design an elliptic IIR filter with order 7 and then design a minimax FIR filter with order 11 . Thus the overall orders are $M$ 
and $N \quad$. Because of the symmetry of the numerator, the number of multiplications in the numerator is 10 . The total number of multiplications is therefore 17. The pulse power spectrum is shown in Fig. 3C. The filling efficiency $\eta \quad \%$ in this case.

The mask-filling efficiencies are summarized in Table I. At the receiver, the frequency component outside . $\mathrm{GHz} \leq f \leq$. $\mathrm{GHz}$ is often filtered. Therefore the other way to compute the efficiency in Eq. (1) is using $F_{\min } . \mathrm{GHz}$ and $F_{\max }$. GHz. That results a different value of efficiency. They are also listed in Table I. The power spectra corresponding to $F_{\min } \quad \mathrm{GHz}$ and $F_{\max } \quad \mathrm{GHz}$ are shown in Fig. 3. Experiments show that the new method has a consistently higher filling efficiency than the FIR based method.

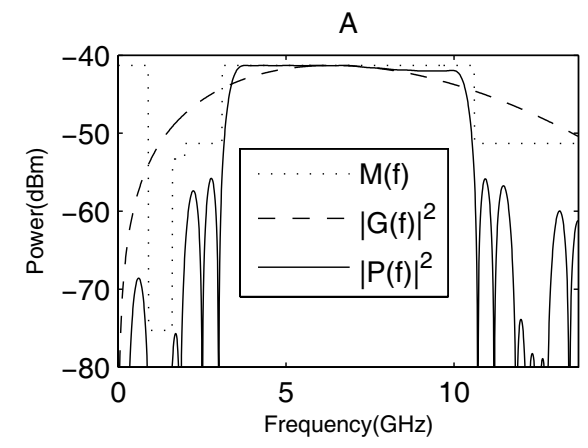

$B$

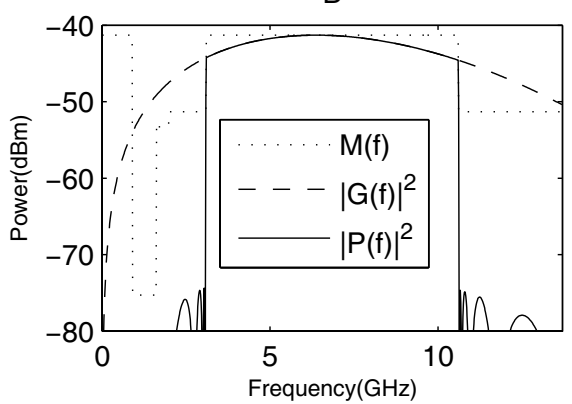

C

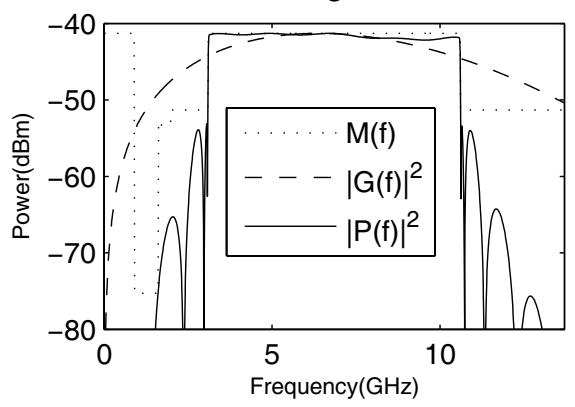

Fig. 3. Results of FIR and IIR approximations to the FCC power mask. (A) FIR based method. (B) IIR elliptic method. (C) The new method of Section

\begin{tabular}{|c|c|c|c|}
\hline & $\mathrm{A}$ & $\mathrm{B}$ & $\mathrm{C}$ \\
\hline $\begin{array}{l}F_{\min }=0 \mathrm{GHz} \\
F_{\max }=13 \mathrm{GHz}\end{array}$ & $74.96 \%$ & $68.29 \%$ & $78.92 \%$ \\
\hline $\begin{array}{l}F_{\min }=3.1 \mathrm{GHz} \\
F_{\max }=10.6 \mathrm{GHz}\end{array}$ & $88.06 \%$ & $80.62 \%$ & $92.61 \%$ \\
\hline
\end{tabular}

TABLE I

MASK-FILLING EFFICIENCY FOR THE THREE DESIGN METHODS.

Transient Response. In UWB transmission, shorter pulse duration results in better transmission rates. Therefore, one must consider the pulse duration when designing the transmitting pulse [5]. By definition, IIR filters have infinite impulse response. That means the pulses generated from Examples $\mathrm{B}$ and $\mathrm{C}$ have infinite duration. However, the experiments show that the impulse responses of these IIR filters decay very fast. The transient is thus negligible. Fig. 4 shows the impulse responses of the shapers for the three methods. The impulse response of the FIR shaper has a duration of . ns. The impulse response of the new method has more than $98 \%$ of its energy within this duration. The rest of the energy (less than $2 \%$ ) can be viewed as transient and is small enough to be neglected. Fig. 5 shows the pulse $p(t)$ for the three cases.
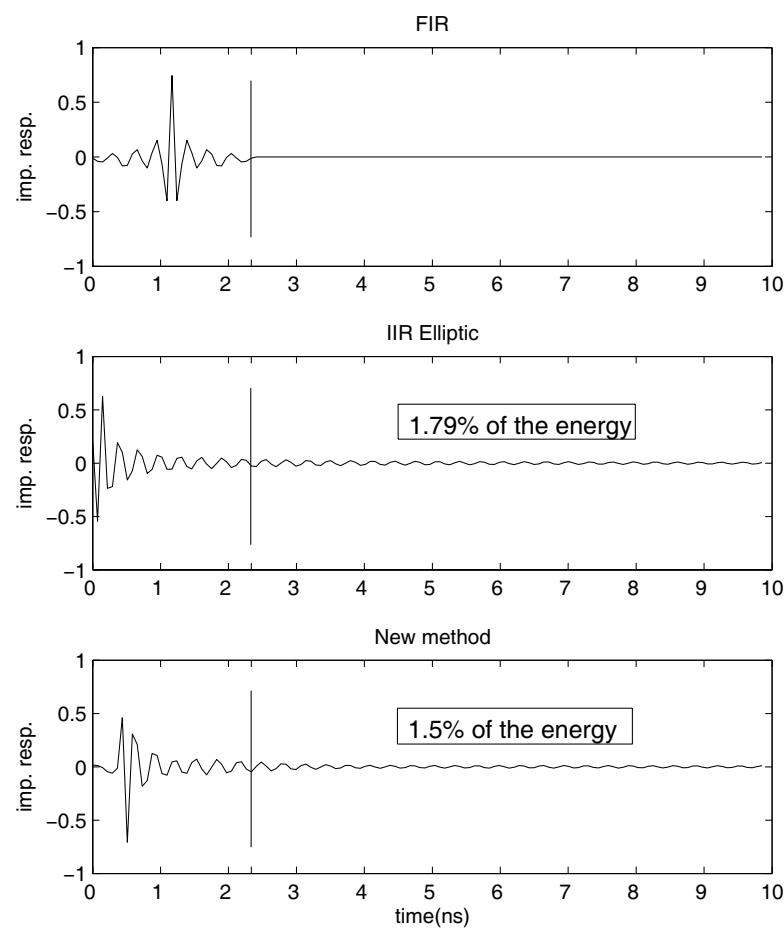

Fig. 4. Impulse responses $h(n T)$ for the three shapers. 

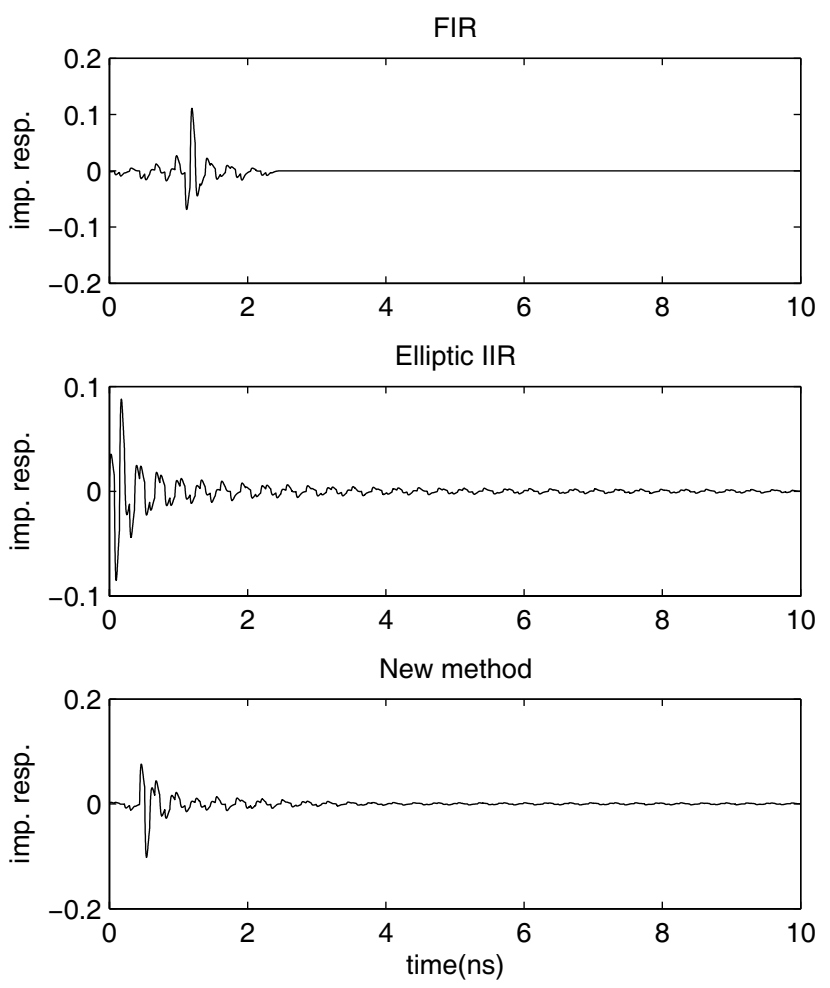

Fig. 5. Pulse $p(t)$ for the three designs.

\section{IMPLEMENTATION DETAILS}

In this section, we further discuss the implementation of the IIR shaper. The number of multiplications of the $N$ th order elliptic IIR filter can be further reduced to $N$ by using the allpass decomposition [7] along with the lattice implementation of the allpass filters if $N$ is odd. For example, Example C in Section IV has a 7th order elliptic filter and an 11th order minimax FIR filter. The number of multiplications of the elliptic filter can be reduced to 7 . Therefore the overall number of multiplications can be reduced to $7+6=13$. Furthermore, using this implementation, the magnitude response in the passband has low sensitivity to quantization of the filter coefficients.

By the allpass decomposition theorem described in [7] Section 3.6, for any $N$ th order elliptic filter $H(z)$, there exists $n_{0}$ th order allpass filter $A_{0}(z)$ and $n_{1}$ th order allpass filter $A_{1}(z)$ such that

$$
H(z) \quad \underline{A_{0}(z)+A_{1}(z)}
$$

and $n_{0}+n_{1} \quad N$. Furthermore, if $N$ is odd, the coefficients of $A_{0}(z)$ and $A_{1}(z)$ are real. By this decomposition, we can implement an elliptic filter with two allpass filters. Moreover, the allpass filters can be implemented by the lattice structure ([7] Section 3.4.3). The number of multiplications for implementing an $n$th order real allpass filter is $n$ if we use the one-multiplier lattice structure. Therefore, the total number of multiplications to implement an $N$ th order elliptic filter is $n_{0}+n_{1} \quad N$, for odd $N$. In Table I, the complexity was 17 multipliers in each of the three cases. For the method C, however, this reduces to 13 multipliers if we use the allpass decomposition method.

The IIR direct form in Fig. 2 is known to be very sensitive to coefficient quantization. In [7] Section 9.7, it is shown that when the magnitude response equals one, the derivatives of the magnitude response with respect to multiplier coefficients are zeros for the lattice structure implementation. This means the magnitude response in the passband is less sensitive to the changes in multiplier coefficients. This is very attractive because the flatness of the passband has a great influence on the efficiency $\eta$. Thus, this implementation not only saves complexity but also has a low sensitivity to quantization.

\section{CONCLUDING REMARKS}

In this paper, an IIR based method for generating the UWB pulse was introduced. An IIR elliptic filter and a minimax FIR filter are combined to get sharp transition bands and flexibility to adjust the passband response. Simulations show that the new method has a better filling efficiency for a fixed complexity, and the transient caused by the IIR shaper is negligible.

\section{REFERENCES}

[1] L. Yang and G.B. Giannakis, "Ultra-wideband communications: an idea whose time has come," IEEE Signal Procesing Magazine, Vol.21, pp.2654, Nov. 2004.

[2] Federal Communications Commission, "Revision of Part 15 of the Commision's rules Regarding Ultra-Wideband Transmission Systems: First report and order," Technical Report FCC 02-48 (adopted Feburary 14, 2002; released April 22, 2002)

[3] Terry P. Lewis, Robert A. Scholtz, "An ultrawideband signal design with power spectral density constraints," Proc. 38th IEEE Asilomar Conf. on Signals, Systems, and Computers, pp. 1521-25, Nov. 2004.

[4] X. Luo., L. Yang, and G.B. Giannakis, "Designing optimal pulse-shapers for ultra-wideband radios," Proc. of IEEE Conf. on Ultra Wideband Systems and Technologies, pp. 349-353, Nov. 2003.

[5] B. Parr, B. Cho, K. Wallace, and Z Ding, "A Novel Ultra-Wideband Pulse Design Algorithm,” IEEE Comm. Letters, pp. 219-221, 2003.

[6] Alan V. Oppenheim, Ronald W. Schafer, Discrete-Time Signal Processing, Prentice Hall 1999.

[7] P.P. Vaidyanathan, Multirate Systems and Filter Banks, Prentice Hall 1993. 